\title{
Urgences
}

\section{Heureux les pauvres}

Denuis Saint-Yves

Numéro 3, 4e trimestre 1981

URI : https://id.erudit.org/iderudit/025048ar

DOI : https://doi.org/10.7202/025048ar

Aller au sommaire du numéro

Éditeur(s)

Urgences

ISSN

0226-9554 (imprimé)

1927-3924 (numérique)

Découvrir la revue

Citer ce document

Saint-Yves, D. (1981). Heureux les pauvres. Urgences, (3), 69-87.

https://doi.org/10.7202/025048ar

Ce document est protégé par la loi sur le droit d'auteur. L'utilisation des services d'Érudit (y compris la reproduction) est assujettie à sa politique d'utilisation que vous pouvez consulter en ligne.

https://apropos.erudit.org/fr/usagers/politique-dutilisation/
Cet article est diffusé et préservé par Érudit.

Érudit est un consortium interuniversitaire sans but lucratif composé de l’Université de Montréal, l'Université Laval et l'Université du Québec à Montréal. Il a pour mission la promotion et la valorisation de la recherche. https://www.erudit.org/fr/ 
DENUIS ST-YVES

Heureux les pauvres 
Pauvre que tu es, désormais tu les détesteras tous autant qu'ils sont ou qu'ils ne sont pas ou qu'ils voudraient être, car cette chose impensable s'est mise à penser par toi, en toi, et cela s'appelle la haine. Et voilà que tu avances vers elle sans en avoir peur, même que c'est peutêtre elle qui a peur de toi car ta haine ne ressemble plus à rien. Elle est pour tout dire sans objet, et pourtant, elle opère de partout en toi, pauvre que tu es. Tu es un rat, et tu n'as plus à te cacher, car tu sens le rat. C'est plus fort que toi, tu détestes, tu es la folie incarnée de la folie et tu deviens l'amoureux permanent de la bête qui te fait plaisir en toi. Tu te parles à la deuxième personne comme si tu étais autrui et que tu pouvais te passer de toi, personnellement parlant! Et qu'est-elle la première personne dont tout te sépare? Cette chose impensable, grotesque, qui s'est infiltrée en toi et en l'autre... la haine. C'est ca la passion au fond de tout. Pleures-tu? 


\section{IX}

Pauvre que tu es, tu voulais devenir un saint, et voilà que tu deviens le sacrifice de la bête. Voilà qu'on ne te regarde plus sur la rue et que tu ne regardes plus la rue. Ce qui est vrai, c'est que tu te détestes presque autant que tu détestes, tout court. Tu es cette blessure qui ne s'ouvre qu'à elle, le ruisseau extraordinaire de ce qui grossit en toi. Le suicide ne veut même pas de toi, car il a peur à la seule pensée que tu le surpasses au niveau de la mort en soi. Tu as ce visage parfait de l'étranger au milieu des autres, même ceux-là qui te connaissent le mieux. C'est d'un coup, d'un seul, que tu as appris que tu ne t'étais jamais habité, habitué, et tu en ris de ce rire indifférent car détaché de toi-même et des choses. Ce savoir te hante, car tu l'as appelé comme on appelle le mal et le mal est trop doux pour ce que tu veux en faire. Pauvre que tu es, la fatigue est ton dernier espoir. 


\section{$X$}

Pauvre que tu es, l'orage ne te donnera jamais qu'un nom de plus, qu'un nom sans phare et tes mains, dis, ce ne sont pas des mains humaines, nécessaires, au sens où généralement on le donne à entendre, mais des armes que tu tends aux autres pour les tirer à toi. Puis la poésie te devient insupportable, elle te tient, elle t'étouffe autant que tu la déshonores à vouloir être poète, pauvre que tu es. Tu ne sais rien à son sujet, dis, et pourtant tu te l'inventes à part dans ta tête, et que les autres se contentent de ton ignorance, de ce rien qui te désarme, qui te rend cruel, et pensif. La poésie te rend semblable à la bête car tu te fermes pour te fermer à la vérité que tu désespères de persuader d'être tienne, pauvre que tu es en tout et en rien. La vérité, ce n'est pas pour toi à la fin du jour, et combien tu le sais au coeur de ta nuit. Ce que tu connais se résume à bien peu d'éléments susceptibles de te sortir de ta haine, car cela se résume à ton front d'enfant dormant sur la table. 


\section{$X I$}

Pauvre que tu es, au fond de tes nuits, tu ne sauras même pas ce qu'aura été ta vie, car elle t'aura ignoré. Ignoré et bafoué, oublié et délaissé tant par l'anarchie que par les solitudes que tu lui auras fait endurer. Tu auras des éclairs flasques de ce qu'aura été ta vie et cela te rassurera dans ta haine. On t'aura mis du vent entre les mains afin que ton mirage s'accomplisse, et tu seras content de tout ton poids de mort. Le drame, c'est qu'il sera une hantise de toi dont tout te séparera, qui te rendra doublement quelconque dans ta haine que tu prendras comme un bâton pour t'achever et te remettre du cauchemar de toi. Ta vie aura été un manque d'air, et tu finiras par mourir car tu ne connais rien de la mort... mais tu feras comme si! 


\section{$X I I$}

Tu n'écris pas, tu t'écris, pauvre que tu es, ignoble dans l'ignoble. De tout ton poids qui n'a rien de mutatif, tu pèses sur les mots en écrivant afin qu'on $y$ reconnaisse ton corps éprouvé éprouvant. Tu te les enfonces profondément dans ta chair de porc malsaine comme des épines abstraites, et quelle croix es-tu capable de porter? Même la plus petite croix qu'on puisse trouver dans l'invisible... tu es indigne de la porter, toi qui te supporte à peine dans le mensonge de toi-même. Tu t'écris sans savoir à qui tu écris, pauvre que tu es. Tu avales des mouches et c'est un spectacle bouleversant que de te voir faire. Si j'étais toi, je m'en irais très très loin en moi, je m'égarerais, je me perdrais, je ne sais... mais tu restes là, comme une relique, une curiosité à la fois drôle et plaintive de la folie, et tu n'en sais rien de la folie, c'est bien ainsi, les fous s'ignorent en eux, entre eux. 


\section{XIII}

Quand tu fermes une porte, c'est l'espace qui brûle derrière toi, pauvre que tu es, et quel est ce pas qui te guette, que tu ne fais pas? Tout est derrière toi, et toi aussi. Ton présent est un passé qui s'ignore et rien n'est moins discutable que ton passé aux claires odes des fontaines où obstinément tu t'es toujours refusé à boire. Et tu pénètres en l'enfant que tu es sans frapper, dis, c'est d'un désordre très noble... tu as au moins le style d'y entrer. Pauvre que tu es, jusqu'où ira ton masque? Même sans masque, tu sembles, tu es masqué et marqué par ton destin et que le hasard s'en mêle n'arrange rien. La seule chose est que ta liberté commence à se faire là... où toi tu commences. 


\section{$X I V$}

Pauvre que tu es, tu te tirerais une balle dans la tête que ce serait une balle utile aux hommes que tu gaspillerais. Tu ne vaux pas la balle à te tuer. Tu es déjà le mort de ce que tu tues autour de toi, dis, cela est suffisant. Tu te prends pour la cible, le tirera du tireur, et te contredire mériterait que tu vives éternellement. Le pont pour toi est double car tu marches sur ses deux rives, et cela est une façon curieuse d'aller au bout de toi-même. Pauvre que tu es, ta haine est si lointaine, si ignoble, qu'il te faut en faire une abstraction jusqu'en toi. 
Il t'arrivera encore de sourire, mais à ce moment-là, tu auras envie de pleurer et de te rouler, seul, dans l'écume des déserts. Tu t'enfonceras en toi jusqu'à perdre raison, et que tu ne puisses plus en sortir à moitié même. Tu auras ce contentement d'ombre que tu es, pauvre... et ton oeil te dira la paupière qui endort comme un paysage à la nuit. Puis, tu prendras le dernier regard, le dernier train, ceux du retard opaque de toi-même, et tu t'en iras à l'autre bout de l'horizon car tu es sa poussière épousant sa poussière. Et le temps, dis, te fera un visage de mort. 


\section{$X V I$}

Pauvre que tu es, voilà que tu pleures, que quelque chose s'est cassé entre toi et les murs de toi, que ta haine n'est plus ta haine, et qu'un moment tout s'efface en toi... II fallait que tu les jettes ces dés du doute et que le plus petit chiffre en sorte et te libère. Pour une fois, la seule en toi à dire vrai, rien n'était truqué, à part toi. Tu te mets à genoux comme pour ouvrir mille fenêtres, et c'est autrui que tu respires à te respirer ailleurs qu'en toi-même. Pauvre que tu es, tu oublies que la bête en toi te guette, prête à bondir sur toi, sur tes pauvretés dans le réel que tu voudrais comprendre si seulement tu avais des mains. Moi et moi, nous t'avons écouté jusqu'au bout pour apprendre que l'homme reste à sauver malgré tout. 


\section{$X V I I$}

Et il advint que la bête sortit de l'homme, s'en alla à la porte de son visage demander un autre asile. 
Toute l'eau de la mer ne suffirait pas à laver une tache de sang intellectuel.

Lautréamont 
Ca commence (par quoi commencer?), l'écriture, la solitude, comme une vitre sur ton front qui tremble du paysage défait et refait en toi. Et que l'écriture soit bonne ou mauvaise ou autre chose, ça ne change rien. Tu ne sais rien pour toutes les autres fois où ton ignorance te faisait passer de bons moments d'érudition fausse. Ce n'est même plus toi qui es seul, banal, quotidien, c'est ta solitude. Elle est sa propre, propre, propre, identité, hors de toi, de tout et de rien. Et dire tu, n'est-ce pas encore de toi qu'il s'agit... à la première personne...? Tu n'en sais rien, rien, rien. Tu ne sais rien et et tu prends rendez-vous avec la connaissance, pour te donner bonne conscience, des raisons à toi d'espérer, pauvre que tu es. Et ce dépassement que tu attends fait de toi le fou dérisoire de la nuit. 
Pauvre que tu es, as-tu jamais regardé un oiseau mourir, comment ses ailes se referment pour s'ouvrir à une autre durée dans laquelle tu fais rire? As-tu jamais seulement regardé tes mains sans nid, pauvre âme que tu es? Le ciel tout entier est cette demeure de l'oiseau qui finit par te... jeter bas, bas, bas. As-tu jamais pensé à une porte sans avoir le goût de pleurer à boire debout...? Tu es le gardien d'un ascenseur dont tu as perdu les clés et dont la seule chose est qu'il monte sans toi à jamais, pauvre que tu es. As-tu seulement encore le droit de penser? TU NAGES et tu CAUSES, pour RIEN, pauvre que tu es et de le dire écoeure celui qui l'entend. Je et toi, c'est pareil... et si j'étais toi je ne voudrais pas être moi. 
Pauvre que tu es, tu ne sauras jamais être toimême, même la nuit, ce qui te fait sourire devant ta propre colère, mièvre et indolente. Devant toi-même et devant l'autre en second lieu, tu n'offriras de toi et de ton devenir qu'une image déformée à laquelle tu tiens plus qu'à toi-même... et c'est peu dire, pauvre que tu es. As-tu regardé, saisi et compris seulement une fois l'heure ailleurs que sur ta montre? Et puis tu te contentes de questionner car les réponses te sont toujours une gratuité difficile à raisonner. Sais-tu seulement que tes chairs s'endormiront pour ne plus avoir à te savoir, croire même qu'elles ont passé en toi? Et tu voudrais comprendre le pourquoi de Prométhée et de sa quête première et éternelle, pauvre que tu es, pauvre tête perdue, mais as-tu seulement regardé une fois tes cendres? 
Et à la fin ta vie n'aura été qu'une longue promenade du lundi, le dimanche étant le regard auquel tu n'as jamais cru, ne sachant, ne voulant rien de l'ordre des choses et de l'être en toi. Tu n'auras que quatre pattes pour te souvenir, et de l'arbre sans fruits qui te rend semblable à la parabole, et surtout de toi-même, pauvre que tu es. Ton moi n'aura rien à dire lorsque la mort et le jugement lui causeront. Pauvre que tu es, ton visage s'en ira tout seul, de lui-même, surtout sans toi. As-tu seulement pensé, ne serait-ce qu'une fois, que le temps est le signe d'un autre temps? De l'amour, celui qui est providence, tu n'auras retenu que la demeure animale, une fin en soi. 
Pauvre que tu es, auras-tu au moins ce courage de mourir réconcilié, comme si ce n'était rien et qu'effectivement ce n'est pas ta perte qui importe, mais ta présence? Tu te nuis à chaque instant de toi-même et il se fait entre toi et le monde une envie de lassitude dont tu es la cause, avec toutes les implications frauduleuses que tu fabriques à partir de rien. Ce rien qui est toi du je au il. Chez toi, la vie est ce pacte de l'agonie au bout d'une nuit sans fin et la peur du rouge à tes poignets d'enfant ironique, et souffrant. Puis l'amour que tu te portes est une offense à l'amour même à cette chose près que tu n'en sais rien. Tu n'aimes pas être seul avec toi-même, alors tu fais semblant de t'aimer, pauvre que tu es. Que connais-tu des sanglots? Rien. Que ce désert soit le tien. 
Pauvre que tu es, tu rêves que le monde peut se passer de toi et même si ce n'est pas toute la vérité, tu te trompes de partout et en toi surtout. Ce n'est pas le monde qui peut, qui veut se passer de toi, mais sa réalité à l'échelle des valeurs que tu ne montes pas avec l'idée d'y rester, mais d'en sortir. Toi au moins la terre te suffit, mais si j'étais la terre, tu n'aurais pour dormir qu'un cadavre. Tiens! tu te fais pitié pour le plaisir de te faire pitié, et moi, cette pitié je me l'emmerde là où tu penses. Et Jésus, en vérité je te le dis, tu l'emmerdes aussi, pauvre que tu es, car tu as entendu sans comprendre le poème. 


\section{VII}

Pauvre que tu es, il te faut cette fois revenir à toi, tu t'évanouis tout seul, sans raison, seul. Tu as peur du noir, seul, et ce noir c'est toimême avec tes lucioles éprouvant le même sentiment que toi. Pauvre que tu es, tu te nourris avec ce que tu ne sèmes pas, attendant je ne sais quel signe des temps. Tu mords à l'illusion comme aux hameçons de l'attente négative, en ce sens que tu ne te verras jamais venir au chemin de la création sans le créer. 